\title{
Surgical reconstruction of semilunar valves in the growing child: Should we mimic the venous valve? A simulation study
}

Peter E. Hammer, PhD, ${ }^{a}$ Erin G. Roberts, MS, ${ }^{a, b}$ Sitaram M. Emani, MD, ${ }^{a}$ and Pedro J. del Nido, MD $^{\mathrm{a}}$

\section{ABSTRACT}

Objectives: Neither heart valve repair methods nor current prostheses can accommodate patient growth. Normal aortic and pulmonary valves have 3 leaflets, and the goal of valve repair and replacement is typically to restore normal 3-leaflet morphology. However, mammalian venous valves have bileaflet morphology and open and close effectively over a wide range of vessel sizes. We propose that they might serve as a model for pediatric heart valve reconstruction and replacement valve design. We explore this concept using computer simulation.

Methods: We use a finite element method to simulate the ability of a reconstructed cardiac semilunar valve to close competently in a growing vessel, comparing a 3-leaflet design with a 2-leaflet design that mimics a venous valve. Three venous valve designs were simulated to begin to explore the parameter space.

Results: Simulations show that for an initial vessel diameter of $12 \mathrm{~mm}$, the venous valve design remains competent as the vessel grows to $20 \mathrm{~mm}(67 \%)$, whereas the normal semilunar design remains competent only to $13 \mathrm{~mm}(8 \%)$. Simulations also suggested that systolic function, estimated as effective orifice area, was not detrimentally affected by the venous valve design, with all 3 venous valve designs exhibiting greater effective orifice area than the semilunar valve design at a given level of vessel growth.

Conclusions: Morphologic features of the venous valve design make it well suited for competent closure over a wide range of vessel sizes, suggesting its use as a model for semilunar valve reconstruction in the growing child. (J Thorac Cardiovasc Surg 2017;153:389-96)

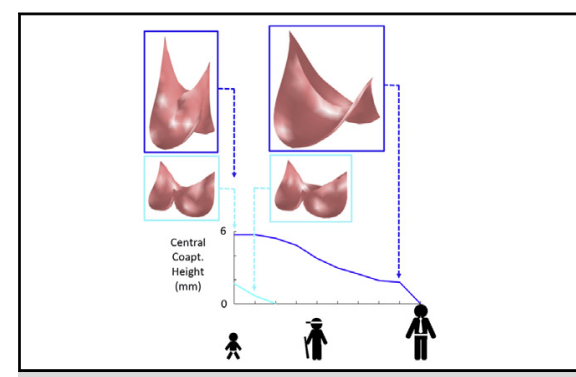

Repair that mimics the $\mathrm{W}$ better accommodates growth than trileaflet repair.

\section{Central Message}

In computer simulation, venous-like valves close over a wide range of vessel sizes. Aortic or pulmonary valve reconstruction that mimics the VV might accommodate growth in the child.

\section{Perspective}

Aortic and pulmonary valve disease in children usually requires multiple interventions because current treatments cannot accommodate patient growth. We recently reported a stented VV in the pulmonary position that can be balloon dilated as the child grows. In the current study, we use simulation to compare 2- and 3-leaflet valve performance during growth. The results suggest that valve repair mimic the VV.

See Editorial Commentary page 396.

See Editorial page 387.
In congenital heart disease, the aortic and pulmonary valves are often affected. When valve disease is moderate to severe, surgery is required to prevent adverse cardiac remodeling that can ultimately progress to heart failure.

\footnotetext{
From the ${ }^{\mathrm{a}}$ Department of Cardiac Surgery, Boston Children's Hospital; and ${ }^{\mathrm{b}}$ Division of Materials Science and Engineering, Boston University, Boston, Mass.

This study was supported by Grant R01 HL110997 from the National Institutes of Health.

Received for publication Jan 27, 2016; revisions received July 26, 2016; accepted for publication Aug 2, 2016; available ahead of print Sept 21, 2016.

Address for reprints: Peter E. Hammer, PhD, Department of Cardiac Surgery, Boston Children's Hospital, 300 Longwood Ave, Boston, MA (E-mail: peter.hammer@ childrens.harvard.edu).

$0022-5223 / \$ 36.00$

Copyright (c) 2016 by The American Association for Thoracic Surgery

http://dx.doi.org/10.1016/j.jtcvs.2016.08.019
}

In children, surgical repair of the valve is usually preferred over valve replacement. ${ }^{1,2}$ In some cases, it is possible to preserve the native vessel and to reconstruct only the leaflets using pericardium or other tissues as grafts. However, the presence of nongrowing leaflet grafts within a growing native vessel ultimately leads to regurgitation, stenosis, or both (Figure 1).

Scanning this QR code will take you to a video for the article.

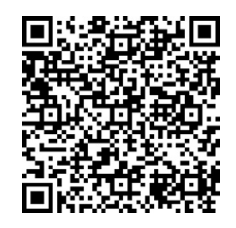




\section{Abbreviations and Acronyms \\ $\mathrm{SV}=$ semilunar valve \\ $\mathrm{VV}=$ venous valve}

In patients in whom valve repair is not possible, the valve must be replaced. In neonates and small children, an aortic or pulmonary valve can be replaced by a homograft from a child donor of appropriate size, although demand for such small homografts greatly exceeds supply. ${ }^{3}$ Another approach is to use an adult-sized homograft that has been downsized by removing one third of the conduit including 1 leaflet. ${ }^{4,5}$ Valved femoral vein homografts also have been used and are available in appropriately small sizes, ${ }^{6}$ as are decellularized valved bovine jugular veins. ${ }^{7}$ In all of these cases, the implanted conduit and valve do not become living host tissues, but rather begin to degenerate and limit growth in the patient.

Venous valves (VVs), present in the lower extremities of humans and other large mammals, are usually bileaflet in structure, and their proportions are much different than those of cardiac semilunar valves (SVs). In the normal $\mathrm{SV}$, the axial length of the valve is approximately half the vessel diameter, whereas in the $\mathrm{VV}$, the axial length of the valve is considerably greater than the vessel diameter (Figure 2). Furthermore, in contrast to the SVs, the free edge of a VV leaflet is considerably longer than the vessel diameter. These shape differences might allow the closed valve to be more robust to vessel diameter changes. In fact, a VV is known to exhibit competent closure across a wide range of local venous pressure and vessel size caused by short-term stresses, such as postural changes and exercise. We hypothesize that on the basis of geometric factors alone, a venous-like valve design can close over a wider range of vessel diameters than a semilunar-like valve design. When the surgical reconstruction of a cardiac SV entails suturing nongrowing leaflet grafts into a preserved outflow tract of a growing child, a reconstruction that mimics the bileaflet $\mathrm{VV}$ might be more resistant to regurgitation as the patient grows, thus delaying the need for reintervention.

To test this hypothesis, we used computer simulation. We have previously used computer simulation to study how different techniques for aortic valve surgical reconstruction affect the robustness of valve closure immediately after surgery. ${ }^{8,9}$ For these studies, the primary advantage of simulation was that it allowed a single dimension or feature of valve reconstruction to be varied while keeping all others constant. In this work, we extended our methods to simulate closure of a reconstructed valve in response to somatic growth, specifically isolating the effect of a VV versus an SV design on the reconstructed valve performance over a range of valve diameters. In this report, we describe our

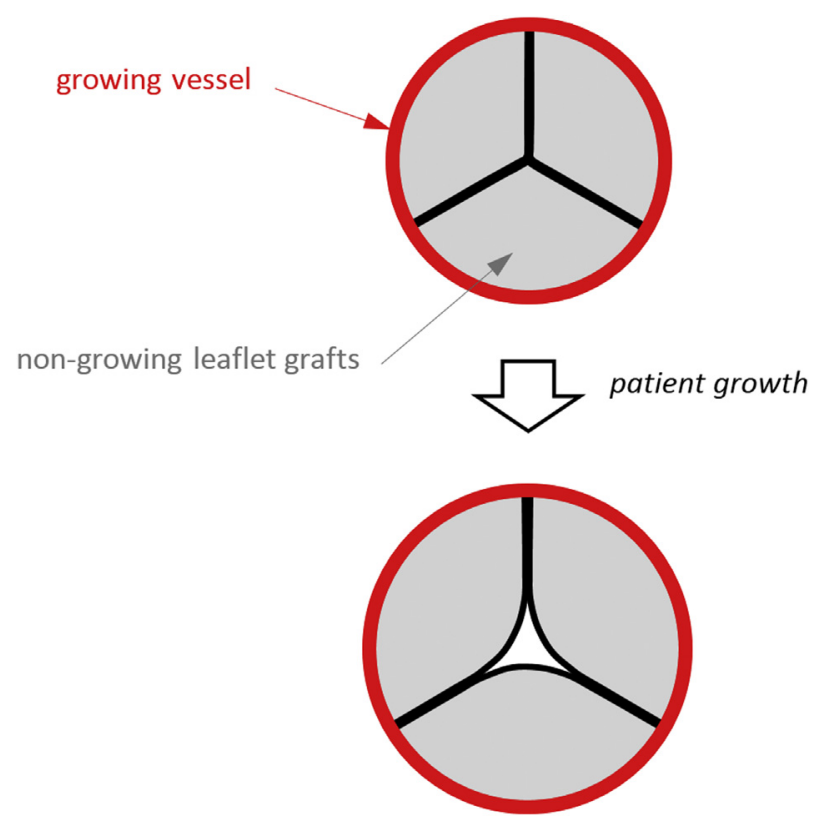

FIGURE 1. Top: Cross-section of a reconstructed SV in the closed position, with preserved (growing) vessel shown in red and leaflet grafts (nongrowing) shown in gray. Bottom: As the vessel grows, the nongrowing leaflet grafts are pulled apart as the vessel (to which the leaflets are attached) moves radially outward with growth.

method for simulating the closed, loaded state of a reconstructed aortic/pulmonary valve and for predicting its competence in response to various degrees of somatic growth. We use the simulated closed states to assess valve competence and to compute measures of interleaflet coaptation. We also compute the effective orifice area of the open valve to estimate outflow resistance during systole.

\section{MATERIALS AND METHODS}

In this section, we describe the methods used to simulate the closed, loaded state of both a VV design and a normal SV design. The geometry of the 2 valve designs is described, as are the mechanical properties of the tissues used for valve reconstruction. We then describe how we simulate valve function in the presence of somatic growth and how diastolic and systolic function of the valve are quantified.

\section{Finite Element Model of Nongrowing Leaflet Grafts in a Growing Vessel}

To compare the amount of growth tolerated by bileaflet, venous-like valves with that of a typical trileaflet, semilunar-like valve, we used a structural finite element model of valve leaflet grafts attached to the walls of a cylindrical vessel of varying diameter. To predict the ability of a reconstructed valve to close without regurgitation during growth, we simulated the closed state of the valve in diastole, the phase of the cardiac cycle when the SVs are pressurized and most likely to leak. Furthermore, although we are interested in the behavior of reconstruction of both aortic and pulmonary valves, we used valve shape data from the aortic valves to define the geometry of the trileaflet, semilunar-like valve, and for both valve morphologies we simulated diastolic loading of the valves using values of systemic (arterial) pressure corresponding to the simulated age of the growing child. 


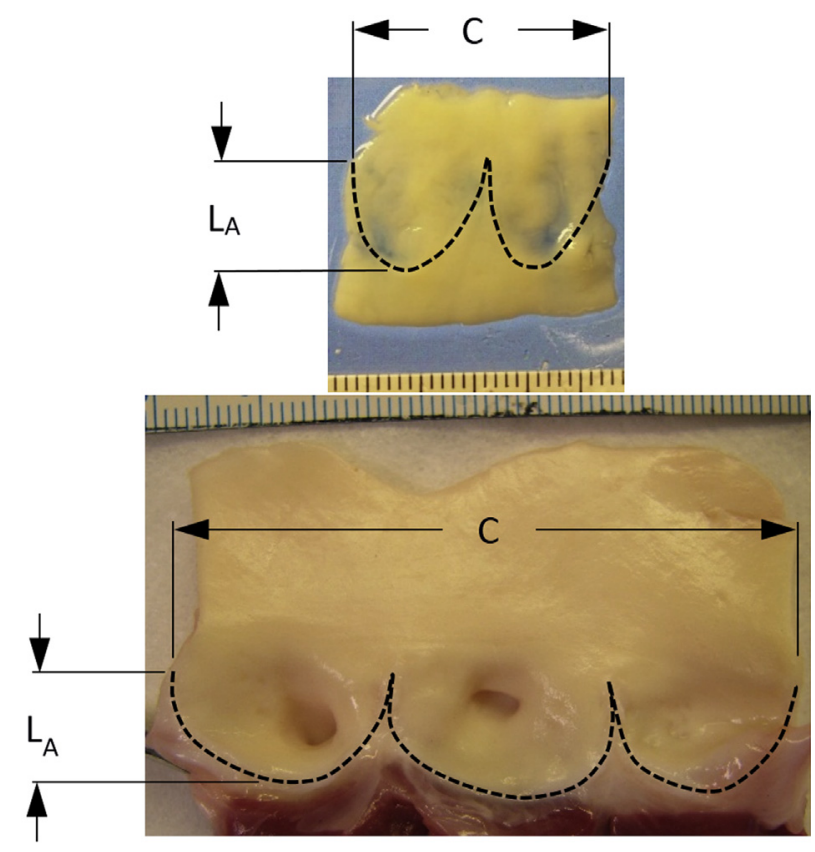

FIGURE 2. Segments of a human femoral vein (top) and porcine aortic valve (bottom) from which the valve leaflets have been excised. Dimension $\mathrm{C}$ indicates the circumference of the vessel at the top of the leaflet attachment, and dimension $\mathrm{L}_{\mathrm{A}}$ indicates the length of the leaflet attachment in the axial direction of the vessel. Scales in both photographs indicate millimeters. In the VV, the axial length of the valve is considerably greater than the vessel diameter (1.3 times), whereas in the aortic valve, the axial length of the valve is approximately equal to half the vessel diameter.

Valve geometry. To create an SV design model, we scaled the average leaflet shape determined from studies of 18 porcine hearts ${ }^{10}$ to the appropriate size for a child with an aortic root of 12-mm diameter. For the shape of the bileaflet, VV design, we took measurements from a single human femoral vein and scaled the resulting leaflet shape to a 12-mm diameter root. The planar leaflet outlines for both designs were meshed with triangles, replicated, and joined at their end points (Figure 3, $A$ and $C$ ). The planar meshes were wrapped into cylinders (Figure 3, $B$ and $D$ ) on the basis of anatomic studies showing that the points of attachment of the leaflets to the aortic root lie on a cylinder ${ }^{11}$ and our own experimental observations in both SVs and VVs. Approximately 300 triangles were used to represent the valve leaflet surface in each modeled valve morphology. To begin to explore the parameter space of the VV design, we ran simulations using 2 additional leaflet shapes. Our planar, excised human femoral vein specimen exhibited a ratio of leaflet midline height to circumferential leaflet width of approximately $40 \%$ (design VV1). We also constructed and simulated valves with this ratio equal to $50 \%$ (VV2) and 60\% (VV3) (Figure 3, E).

Leaflet mechanical properties. The finite element method that we applied uses knowledge of the relationship between stress and strain in a material to simulate deformation of the structures in response to applied loads. Heart valve leaflets and materials typically used for valve reconstruction undergo relatively large deformations under physiologic loads, and this requires a description of the material mechanical properties appropriate for finite deformation theory. Accordingly, we describe leaflet mechanical properties using an exponential strain energy function of the form:

$$
W=\frac{c}{2}\left(e^{Q}-1\right)
$$

where $W$ is strain energy density, $c$ is a constant and

$$
Q=A_{1} E_{11}^{2}+A_{2} E_{22}^{2}+2 A_{3} E_{11} E_{22}+A_{4} E_{12}^{2} .
$$

The values of $A$ are constants, and $E_{11}, E_{22}$, and $E_{12}$ are the normal and shear components of the Green strain tensor. Components of the second Piola-Kirchhoff stress tensor are computed as the partial derivatives of $W$ with respect to the respective components of the Green strain tensor. The values of parameters $c$ and $A$ were chosen not to explicitly describe $\mathrm{VV}$ leaflets of aortic or pulmonary valve leaflets, but rather to approximate general distensible tissue suitable for valve reconstruction $(c=50 \mathrm{kPa}$, $A_{1}=4, A_{2}=4, A_{3}=0, A_{4}=4$ ). The leaflets are modeled as a mesh of triangular membrane elements with a constant thickness of $0.5 \mathrm{~mm}$.

Simulating the pressurized valve in the growing root. We simulated the state of the valve under diastolic pressure in 2 steps. First the vessel, modeled implicitly as an elastic cylinder serving as the boundary to which the leaflets attach, was dilated to reach a target level of patient growth. The meshes representing the leaflet grafts do not grow and in fact may constrain the vessel during growth. To achieve a dilated state of the mesh boundary points that is consistent with the physical problem, we applied circumferential and axial forces to the boundary points representing stresses in the dilated vessel for a given diameter and pressure, and we also applied nodal forces due to stresses in the leaflets in elements that have an edge on the cylindrical boundary. ${ }^{9}$ From this condition representing the pressurized aortic root, the locations of mesh boundary points are held constant while surface normal forces representing transleaflet diastolic pressure are applied to close and load the leaflets. The closed state of the valve at normal peak pressure was computed by solving for the equilibrium position of the leaflets using the equations of motion (Newton's Second Law), where forces act on the vertices of mesh triangles due to transleaflet pressure, leaflet deformation, inertial forces, and interleaflet contact. To estimate the forces due to leaflet deformation, we used a finite element approach developed for materials that undergo large deformations. ${ }^{12}$ The equations of motion were discretized using a second-order backward difference method and were solved using semiimplicit numeric integration with adaptive step size control. Simulation and analysis software were written in the MATLAB programming language (MathWorks, Natick, Mass). The structural finite element analysis method that we have developed and applied was validated in previous work using simulated biaxial loading of square patches of leaflet tissue ${ }^{13}$ and ex vivo experiments of pressurized isolated aortic valves. ${ }^{8}$ Our previous work demonstrates the details of the simulation methods. ${ }^{9}$

\section{Simulating Growth}

In the growing child, the diameter of the aortic root increases from approximately $10 \mathrm{~mm}$ in the neonate to more than $20 \mathrm{~mm}$ in the young adult. ${ }^{14}$ We first simulated closure of a valve within a root with a diameter in diastole of $12 \mathrm{~mm}$, representing a pediatric patient of approximately 2 years of age. Leaflet size was chosen so that leaflets were in the unstressed state when incorporated into the $12-\mathrm{mm}$ diameter vessel. The closed state of the valve was simulated for the $12-\mathrm{mm}$ diameter vessel size and at $1-\mathrm{mm}$ increments in vessel size until the leaflets failed to meet in the valve center. Only the leaflet boundary, representing the preserved growing vessel, changed in size between simulations, whereas the leaflet mesh, representing the nongrowing leaflet grafts, remained a fixed size.

\section{Quantifying Valve Closure}

The robustness of closure of the valve was quantified by the amount of overlap of the leaflets in the valve center under peak load. Surgeons have noted that in the center of the valve, the amount of overlap, referred to as "central coaptation height," for a normal aortic valve is 3 to $5 \mathrm{~mm}$ and that successful aortic valve reconstruction is dependent on a large central coaptation height. ${ }^{15}$ From simulations of the pressurized valve, we 


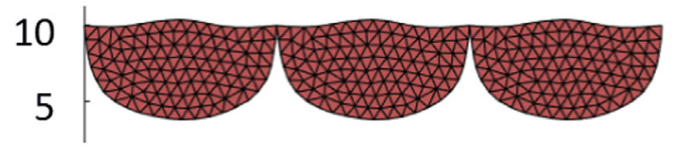

A

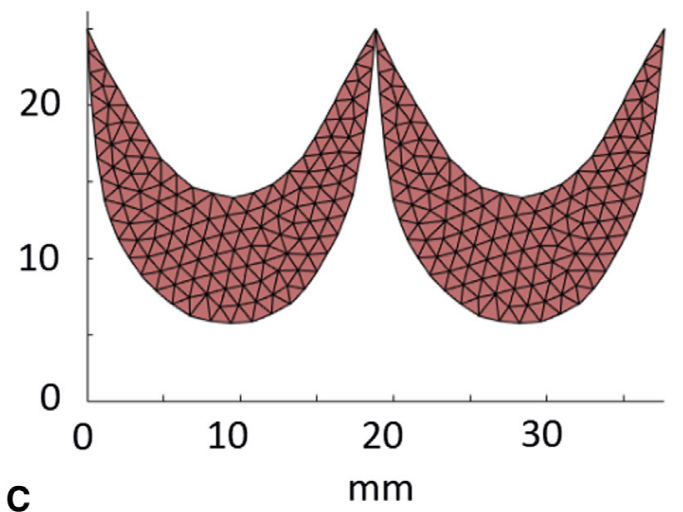

B

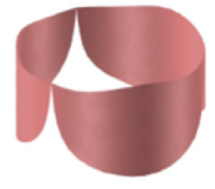

D

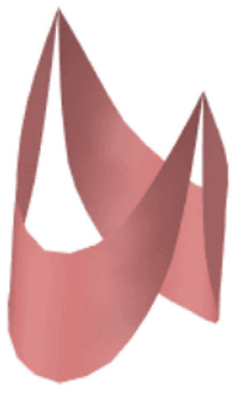

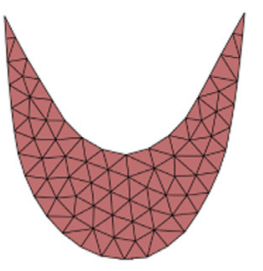

E

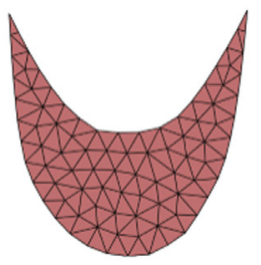

VV2

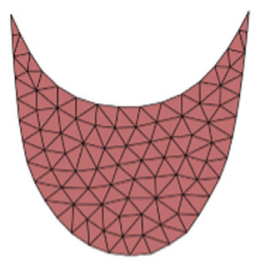

VV3

FIGURE 3. A, Planar outlines of the leaflets from an SV design were meshed with triangles and replicated. B, Planar leaflet mesh was then wrapped into a cylinder with diameter of $12 \mathrm{~mm}$. C, Planar outlines of the leaflets from a VV design were meshed with triangles and replicated. D, Planar leaflet mesh was then wrapped into a cylinder with a diameter of $12 \mathrm{~mm}$. E, Three different VV leaflet designs were tested: VV1 with a midline height to overall height ratio of approximately 0.4 (based on our human femoral vein valve specimen), VV2 with a height ratio of 0.5 , and VV3 with a height ratio of 0.6 . $V V$, Venous valve.

computed the region of interleaflet contact, calculating both its area and the axial height of this region in the valve center.

\section{Quantifying Valve Opening}

The extent to which the simulated valve opens completely during systole was approximated by applying a $3 \mathrm{~mm} \mathrm{Hg}$ pressure difference across each leaflet element in the direction that opens the valve. The effective valve orifice area was then computed as the area of the open valve projected onto a plane perpendicular to the valve axis. Normalized area was then calculated as the effective valve orifice area divided by the cross-sectional area of the vessel.

\section{RESULTS}

Inspection of the simulated closed state of the reconstructed valve showed that the VV design based on the human femoral valve shape (VV1) maintained competent closure during vessel growth up to a maximum vessel diameter of $16 \mathrm{~mm}$ (Figure 4, A). The VV designs with progressively greater leaflet midline dimensions (VV2 and VV3) maintained competent closure up to maximum vessel diameters of 19 and $20 \mathrm{~mm}$, respectively (Figure $4, B$ and $C$ ). On the other hand, the SV design maintained competent closure only to a vessel diameter of $13 \mathrm{~mm}$ (Figure 4,D). Under diastolic pressure load, design VV1 is competent (central coaptation height $>0$ ) as the vessel grows from the initial vessel diameter of $12 \mathrm{~mm}$ to a diameter of $16 \mathrm{~mm}$ (Figure 5, A). With further increases in vessel diameter, central coaptation vanished and the leaflets opened in the valve center, corresponding to central regurgitation of blood. Designs VV2 and VV3, with progressively greater leaflet midline dimensions, maintained central leaflet coaptation to vessel diameters of 19 and $20 \mathrm{~mm}$, respectively (Figure 5, A). The SV design was less robust to vessel growth, maintaining central leaflet coaptation to a vessel diameter of only $13 \mathrm{~mm}$. A similar trend was seen in the area of leaflet coaptation, with coaptation area of the SV design leaflet decreasing more sharply than that of the $\mathrm{VV}$ designs as vessel diameter grew to values greater than $14 \mathrm{~mm}$ (Figure 5, B).

The results of our simplified estimate of valve opening show that all 4 valve designs exhibited a normalized effective orifice area of approximately 1.0 at the initial size of the vessel $(12 \mathrm{~mm})$, indicating that the valves open 

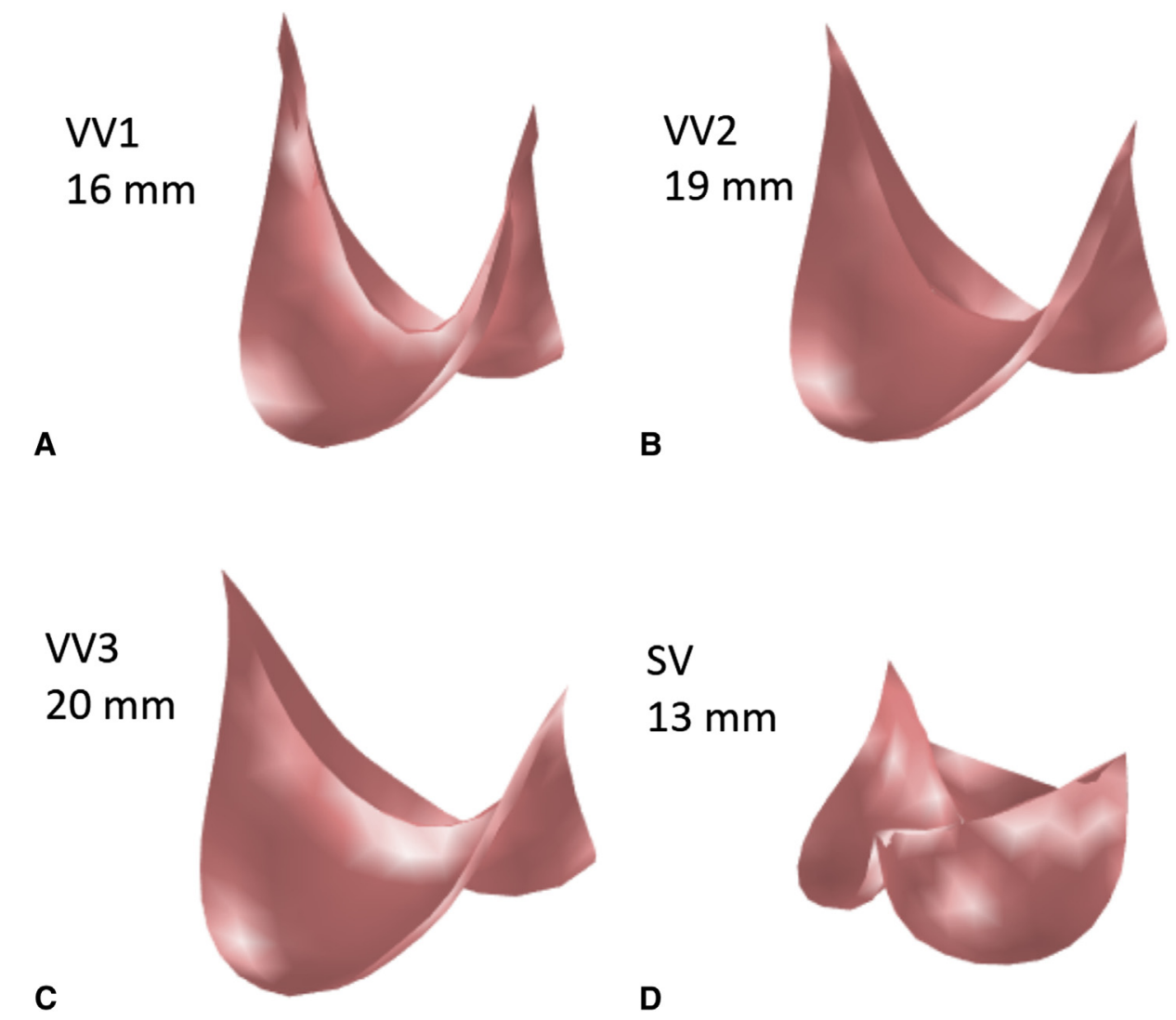

FIGURE 4. Results of simulations showing the loaded state of the reconstructed valve after the maximum vessel growth under which the valve remained competent. A, VV1 design at vessel diameter of $16 \mathrm{~mm}$. B, VV2 design at a vessel diameter of $19 \mathrm{~mm}$. C, VV3 design at a vessel diameter of $20 \mathrm{~mm}$. D, $S V$ design at a vessel diameter of $13 \mathrm{~mm}$. $V V$, Venous valve; $S V$, semilunar valve.

completely (Figure 6). Normalized area decreases with increasing vessel diameter for all 4 designs. The VV1 design exhibits the slowest rate of area decrease with vessel diameter, followed by the VV2, VV3, and SV. Area data could not be computed for the SV design at vessel diameters more than $15 \mathrm{~mm}$ because it was not possible for the simulated vessel diameter to grow beyond that point because of the constraint imposed by the nongrowing leaflets.

\section{DISCUSSION}

The goal of this simulation study was to compare the capacities of 2 different valve morphologies, an SV shape and a VV shape, to accommodate vessel growth in the absence of leaflet growth in pediatric patients. The results suggest that there are inherent, geometric features of the VV design, with its bileaflet morphology and relatively tall leaflet attachments, that allow it to close over a wider range of vessel sizes than the normal SV design. One salient feature that differentiates the 2 morphologies is the length of the free edge of the leaflet. This free edge length imposes a theoretic limit to how large a vessel the leaflet can accommodate because the free edge must extend from the vessel wall to the valve center and back during diastole for complete closure. Thus, it is impossible for a leaflet with a free edge less than twice the vessel radius to close completely. In the normal SV, the leaflet free edge is only slightly longer than twice the radius of the valve root in diastole, whereas in the VV design, the leaflet free edge is more than 4 times the vessel radius in diastole, allowing it to span the distance to the valve center at greater vessel sizes. A second salient feature of the VV design is the bileaflet morphology. For a bileaflet valve, there are 2 interleaflet commissures, approximately 180 degrees apart, that are pulled in opposite directions as the vessel grows. As a consequence, the midpoint of a leaflet free edge moves axially upward along the valve center in response to vessel growth. For a trileaflet valve, there are 3 interleaflet commissures, spaced approximately 120 degrees apart around the vessel wall. In this case, as vessel growth moves the commissures radially outward from the vessel axis, the midpoint of a leaflet free edge is pulled both axially upward and radially outward. The combined effect of these 2 features of the VV morphology can be seen in our simulation results, with the best case VV design (VV3) remaining competent through $8 \mathrm{~mm}(67 \%)$ of vessel growth, whereas the SV design remained competent through only $1 \mathrm{~mm}(8 \%)$. 


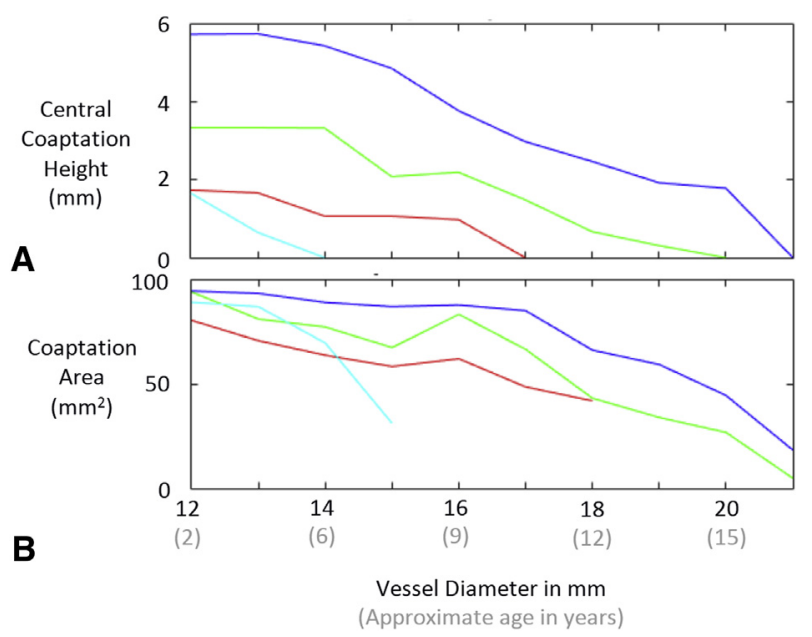

FIGURE 5. Results of simulations showing central coaptation height (A) and coaptation area (B) versus vessel diameter for 4 different valve reconstruction designs: SV (light blue), VV1 (red), VV2 (green), and VV3 (dark blue).

This result could have important clinical implications and suggests that a valve reconstructed with semilunar morphology in a 2-year-old child will begin to exhibit central regurgitation at approximately 4 to 6 years of age, whereas with a VV morphology, the valve could remain competent until 15 or 16 years of age (Figure 5).

In current surgical practice, 3-leaflet SVs are usually reconstructed in a way that maintains normal SV morphology. However, there is precedent for exploiting the VV properties in cardiac surgery. Our group has implanted stented bovine jugular vein grafts (Melody valve; Medtronic Inc, Minneapolis, Minn) in the mitral position in infants and children and showed that the valve can subsequently be expanded via balloon catheter as the child grows, while maintaining competence. ${ }^{16}$ However, it is worth noting that the Melody valve is a 3-leaflet VV, and it is not known whether it can accommodate the same degree of growth as the bileaflet VV design.

An important variable that we did not explore is the relative stretch between the vessel and the leaflet graft at the time of implantation. Our simulations assume that the attachment of leaflet graft is unstretched when the diastolic diameter of the vessel is $12 \mathrm{~mm}$. To achieve this, the surgeon incorporates redundancy into the graft using a running suture that progresses more (ie, larger bites) on the graft than on the vessel wall. ${ }^{17,18}$ It is possible that further increases in this redundancy can produce even greater growth accommodation than in the simulations presented in this article.

Simulation results indicate that with increasing VV leaflet graft height, the amount of growth that the reconstructed valve can accommodate increases, and for a given vessel size, taller grafts lead to better coaptation metrics.

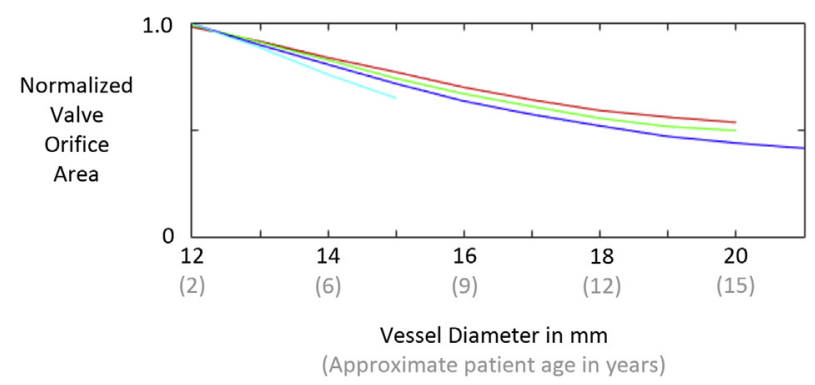

FIGURE 6. Results of simulations that estimate the ability of the reconstructed valve to open during systole as the patient/vessel grows. Normalized effective orifice area is computed by dividing the projected area of the open valve by the vessel cross-sectional area. The curves represent the SV design (light blue) and the $3 \mathrm{VV}$ designs: VV1 (red), VV2, (green), and VV3 (dark blue).

However, tall grafts raise some concerns. First, as the height of the grafts that we simulated increased, the length of the free edge decreased. We surmised that valves with shorter leaflet free edges would become obstructive (small effective orifice area) at earlier stages of vessel growth. This trend can be seen in our results (Figure 6), but it is difficult to know if this difference would be clinically significant. It is also interesting to note that even the tallest VV design (VV3) is associated with a greater effective orifice area than the SV design at a given vessel diameter. A second concern with tall leaflet grafts (eg, design VV3), specifically for aortic valve reconstruction, is that if a leaflet does not close during diastole, it could obstruct flow into the coronary ostia. Further study is needed to understand the effects of these valve design factors.

The data from this simulation can be used to guide techniques for valve repair or to design a prosthesis for valve replacement in children. Guided by simulations, an expandable prosthesis can be designed consisting of a bileaflet valve within an expandable stent with relatively high posts. The results of this simulation are consistent with experimental results from an expandable prosthetic valve using human femoral vein grafts. ${ }^{19}$ Of note, most repair techniques for aortic and pulmonary reconstruction recapitulate semilunar design, which may not retain competence as the surrounding vessel grows. A bileaflet design with tall leaflet attachments may be a better repair strategy given the findings of this study (Video 1 ).

\section{Study Limitations}

Many simplifications were made in this simulation-based study. We simulated a single, generalized graft tissue with isotropic mechanical properties, yet the range of properties of potential graft tissues varies widely because of both biological variability and variable treatment protocols for decellularizing and stabilizing the graft tissues. ${ }^{20,21} \mathrm{We}$ also made no attempt to simulate the complex effects of 


\section{Simulation Results $\quad \begin{gathered}\text { Semilunar design is } \\ \text { incompetent tat } 2 \text { mm of }\end{gathered}$ vessel diameter growth.}

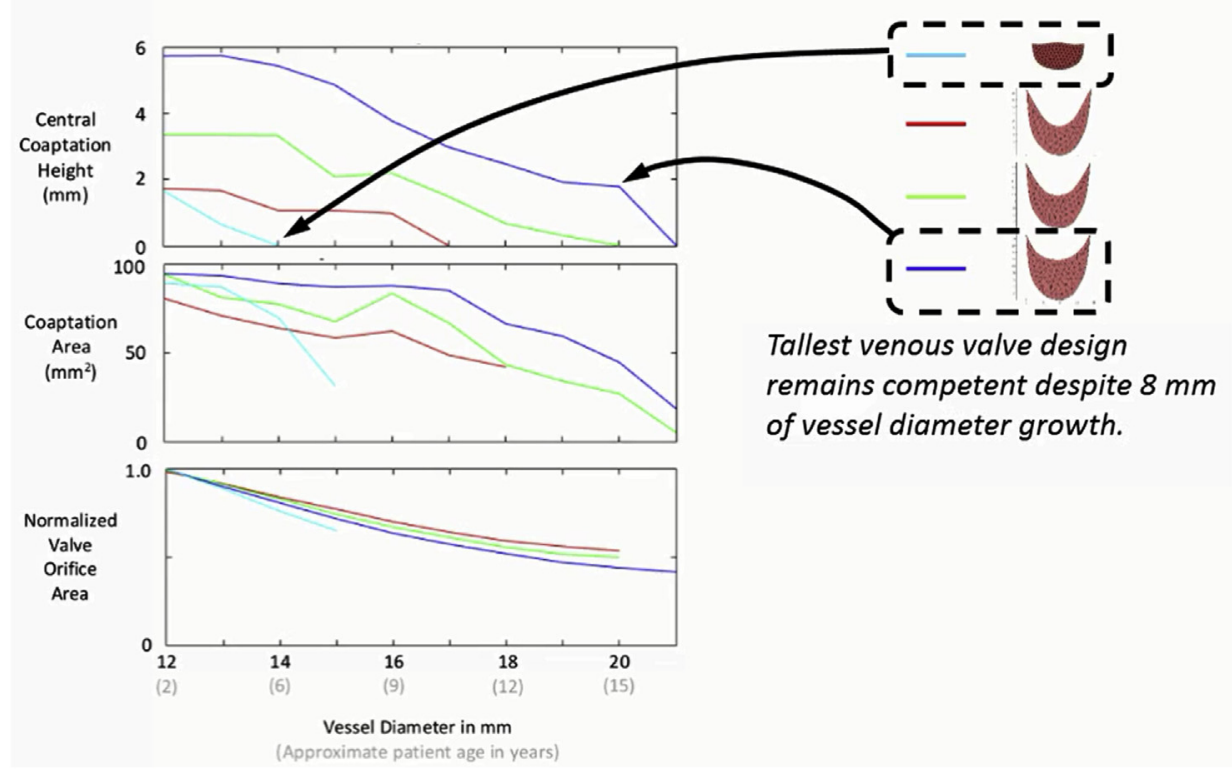

VIDEO 1. In this video, we briefly state the motivation for the study and describe the simulation methods. Two example simulations are shown: one for a reconstruction mimicking a normal SV and one for a reconstruction mimicking a VV. The results of the simulation study are summarized. Video available at http://www.jtcvsonline.org/article/S0022-5223(16)31038-8/addons.

systolic blood flow on leaflet stresses. Furthermore, we neglected to consider biological effects, such as fibrosis, calcification, and retraction, which are known to affect the long-term durability of implanted graft tissues. Another limitation of our study is the relatively large size of mesh elements $(\sim 1 \mathrm{~mm})$ with respect to the output measure of central coaptation height of the valve. This relatively coarse mesh was chosen for computational speed and stability but at the expense of resolution. Smaller mesh elements, particularly near the center of the closed valve, would allow finer resolution of the onset of incompetence with increasing root diameter.

\section{CONCLUSIONS}

Although the results of this simulation study are promising, more work is necessary before this valve reconstruction approach can be brought to the clinic. Our results showed that a venous-like valve reconstruction in a semilunar position has the potential to accommodate patient growth, but we did not thoroughly explore the design space across a wide range of leaflet shapes and material properties to propose an "optimal" design, although such a simulation-based optimization seems feasible. Beyond simulation, carefully designed experiments in explanted tissues will be necessary to compare performance of the reconstruction strategies with real graft materials and under realistic flow conditions for both pulmonary and systemic positions, and in vivo animal procedures will be necessary to predict the biological responses to the proposed valve reconstruction strategy after implantation in the growing child.

\section{Conflict of Interest Statement}

Authors have nothing to disclose with regard to commercial support.

\section{References}

1. Baird CW, del Nido PJ. Complex aortic valve disease in children. Oper Tech Thorac Cardiovasc Surg. 2009;14:253-63.

2. Jonas RA. Aortic valve repair for congenital and balloon-induced aortic regurgitation. Pediatr Card Surg Annu. 2010;13:60-5.

3. Sinha P, Moulick A, Jonas RA. Femoral vein homograft for neoaortic reconstruction in Norwood stage 1 operation. Ann Thorac Surg. 2009;87:1309-10.

4. Michler RE, Chen JM, Quaegebeur JM. Novel technique for extending the use of allografts in cardiac operations. Ann Thorac Surg. 1994;57:83-7.

5. Perri G, Polito A, Gandolfo F, Albanese SB, Carotti A. Outcome of standard and bicuspidalized cryopreserved homografts for primary right ventricular outflow tract reconstruction. J Heart Valve Dis. 2015;24:83-8.

6. Sinha P, Talwar S, Moulick A, Jonas RA. Right ventricular outflow tract reconstruction using a valved femoral vein homograft. J Thorac Cardiovasc Surg. 2010;139:226-8.

7. Hasan BS, McElhinney DB, Brown DW, Cheatham JP, Vincent JA, Hellenbrand WE, et al. Short-term performance of the transcatheter Melody valve in high-pressure hemodynamic environments in the pulmonary and systemic circulations. Circulation. 2011;4:615-20. 
8. Hammer PE, Chen PC, del Nido PJ, Howe RD. Computational model of aortic valve surgical repair using grafted pericardium. J Biomech. 2012;45: 1199-204.

9. Hammer PE, Berra I, del Nido PJ. Surgical repair of congenital aortic regurgitation by aortic root reduction: a finite element study. J Biomech. 2015;48:3883-9.

10. Hammer PE, Pacak CA, Howe RD, del Nido PJ. Straightening of curved pattern of collagen fibers under load controls aortic valve shape. J Biomech. 2014;47:341-6.

11. Swanson WM, Clark RE. Dimensions and geometric relationships of the human aortic valve as a function of pressure. Circ Res. 1974;35:871-82.

12. Taylor RL, Oñate E, Ubach P. Finite element analysis of membrane structures. In: Oñate E, Kröplin G, eds. Textile Composites and Inflatable Structures. The Netherlands: Springer; 2005:47-68.

13. Hammer PE, Sacks MS, del Nido PJ, Howe RD. Mass-spring model for simulation of heart valve tissue mechanical behavior. Ann Biomed Eng. 2011;39:1668-79.

14. Sluysmans T, Colan SD. Theoretical and empirical derivation of cardiovascular allometric relationships in children. J Appl Physiol. 2005;99:445-57.

15. Augoustides JG, Szeto WY, Bavaria JE. Advances in aortic valve repair. J Cardiothorac Vasc Anesth. 2010;24:1016-20.

16. Quinonez LG, Breitbart R, Tworetsky W, Lock JE, Marshall AC, Emani SM. Stented bovine jugular vein graft (Melody valve) for surgical mitral valve replacement in infants and children. J Thorac Cardiovasc Surg. 2014;148 1443-9.

17. Hosseinpour A-R, González-Calle A, Adsuar-Gómez A, Santos-deSoto J. A simple method of aortic valve reconstruction with fixed pericardium in children. Interact CardioVasc Thorac Surg. 2013;16:695-7.

18. Ozaki S, Kawase I, Yamashita H, Uchida S, Nozawa Y, Takatoh M, et al. A total of 404 cases of aortic valve reconstruction with glutaraldehyde-treated autologous pericardium. J Thorac Cardiovasc Surg. 2014;147:301-6.

19. Roberts EG, Quinonez L, Piekarski B, Baird CW, Emani SM. Expandable valve for pediatric application constructed from human venous valved conduit within a stent. Ann Thorac Surg. 2015;100:2320-4.

20. Sacks MS, Chuong CJ. Orthotropic mechanical properties of chemically treated bovine pericardium. Ann Biomed Eng. 1998;26:892-902.

21. Meuris B, Ozaki S, Neethling W, De Vleeschauwer S, Verbeken E, Rhodes D, et al. Tri-leaflet aortic valve reconstruction with a decellularized pericardial patch in a sheep model. J Thorac Cardiovasc Surg. 2016;152:1167-74.

Key Words: venous valve, accommodate growth, semilunar valve, simulation, reconstruction, repair

\title{
EDITORIAL COMMENTARY
}

\section{Design, dynamism, and valve repair}

\author{
John M. Karamichalis, MD, FACS, ${ }^{\text {a,b }}$ Heba Aguib, Dr-Ing, ${ }^{\mathrm{c}}$ Alexandra Anastasopulos, MD, and \\ Magdi Yacoub, FRS, OM ${ }^{\mathrm{c}, \mathrm{d}}$
}

\footnotetext{
From the a Department of Pediatric Cardiac Surgery, The Children's Hospital at Saint Francis, Tulsa, Okla; the ${ }^{b}$ University of California San Francisco, San Francisco, Calif; the ${ }^{\mathrm{c}}$ Biomedical Engineering and Innovation Laboratory, Aswan Heart Centre, Magdi Yacoub Foundation, Aswan, Egypt; and the ${ }^{\mathrm{d}}$ Harefield Heart Science Centre, National Heart \& Lung Institute, Imperial College, London, United Kingdom.

Disclosures: Authors have nothing to disclose with regard to commercial support.

Received for publication Oct 7, 2016; revisions received Oct 7, 2016; accepted for publication Oct 11, 2016; available ahead of print Nov 12, 2016.

Address for reprints: John M. Karamichalis, MD, FACS, The Children's Hospital at Saint Francis, Pediatric Cardiac Surgery, 6161 S Yale Ave, Tulsa, OK 74136 (E-mail: john.karamichalis@ucsf.edu).

J Thorac Cardiovasc Surg 2017;153:396-8

0022-5223/\$36.00

Copyright (c) 2016 by The American Association for Thoracic Surgery

http://dx.doi.org/10.1016/j.jtcvs.2016.10.010
}

One of the most constant aspects of biology is that nothing is constant. This concept is called dynamism, and it is almost ubiquitous from atoms to organs, operating at a wide range of time domains and importantly influencing function. Heart valves are no exception and derive most of their finer functions as well as their durability from the dynamic behavior of their component parts. ${ }^{1}$ Valve dynamism may be active or passive. ${ }^{2}$ The latter is dependent on the design ${ }^{3}$ and the material characteristics of the tissue. Defining these characteristics can be of value in tissue engineering and valve repair, including valve-

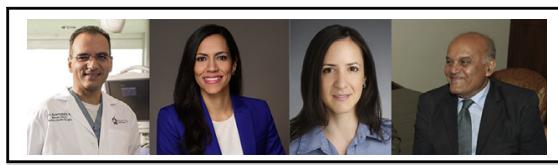

John M. Karamichalis, MD, FACS, Heba Aguib, Dr-Ing, Alexandra Anastasopulos, MD, and Sir Magdi Yacoub, FRS, OM

\section{Central Message}

Heart valves derive their finer function from the dynamic behavior of their component parts.

conserving operations for aneurysms of the aortic $\operatorname{root}^{4}$ (Figure 1). The study of their design characteristics in simulation models can optimize the results of valve repair.

\section{See Article page 389.}

The article in this issue of the Journal by Hammer and colleagues ${ }^{5}$ is a welcome addition to the literature, because it provides an in depth comparison between the design of venous valves and cardiac outlet valves with regard to the number of cusps; their size, shape, and length of attachment to the vessel wall; and also the influence of these designs on the function of the valve when the size of the vessel is changed in a simulation model. 